of Willard Gibbs, provision has been made, through the generosity of a donor who prefers to remain anonymous, for a new and complete edition of Willard Gibbs's writings. This will consist of either two or three volumes, well printed and bound, and will be sold at a very moderate price to encourage a wide distribution. It will probably be published during 1928.

In addition to this reprinting of the original text of Gibbs's works, it is proposed to publish, at some later date, a volume or volumes designed to aid the reader to bridge the well-recognised gap between Gibbs's theorems on one hand, and the actual experimental data of the chemist and physicist on the other. This supplementary material, to be written by competent authorities in the several fields, would aim $(a)$ to explain the philosophical background of Gibbs's method ; $(b)$ to amplify the treatment of points of special difficulty ; $(c)$ to discuss the evaluation of Gibbs's functions in terms of directly measurable quantities; and $(d)$ to furnish a variety of illustrative examples from the literature now available. Such treatment is most needed in the case of the thermodynamic papers, but the plan may be extended to cover Gibbs's writings on other subjects if it seems expedient. The financial support of the undertaking has been liberally provided for, and suitable honoraria will be paid to the authors of the new material.

The undersigned committee, appointed to study this plan, earnestly solicits suggestions and comments from all persons interested, especially with respect to any or all of the following questions :

1. Which of the aims outlined above are the most important ?

2. How should the subject matter be subdivided into parts which can be handled by a single author?

3. What persons, irrespective of nationality, are best fitted by ability and training to undertake these different parts ?

Letters containing suggestions or criticisms will be welcomed, and may be addressed to the Gibbs Committee, Sterling Chemistry Laboratory, New Haven, Conn.

Yale University

JOHN JOHNSTON.

Willitam F. G. Swann.

RALPH G. VAN NAME, Chairman.

New Haven, Conn.

\section{Use of Diffraction Effects in Measurements of Stellar Photographs.}

THE central area of the image of a star in stellar photographs consists of a cluster of silver granules disposed radially in rapidly decreasing numbers, so that the circular or nearly circular boundary is ill defined. This want of definition is necessarily responsible for a large part of the probable error in the microscopical measurements of the star's co-ordinates.

The images of the brightest stars in some photographs, however, may be observed to be accompanied by radial ' rays,' which proceed outwards from both ends of a diameter of the disc as a narrow band gradually terminating in a vanishingly thin line. These effects are well known to be due to diffraction, and it appears that this occasional defect in the image might be purposely produced and used to increase the accuracy of measurement.

If a thin wire be stretched across the aperture of the object glass of the telescope, a star image when observed with an eyepiece will be crossed by a series of narrow spectra at right angles to the direction of the wire, and presumably the central line of this narrow band of interrupted light passes through the point of maximum intensity in the star image. The closer the wire is to the eyepiece the shorter the spectra become, and finally they vanish when the wire coincides with the image. The same narrow band of light is produced when the wire is placed outside the telescope between the object glass and source.

The effect is not altered by moving the wire in a direction perpendicular to the optic axis, and is still apparent when the wire is just within the boundary of the refracted bundle of light; and therefore the presence of other wires parallel to the first only increases the amount of light diffracted into the narrow band of spectra.

It is obvious that if two gratings be constructed of parallel thin wires and crossed at right angles, the images of stars photographed through them will be crossed by two narrow lines at right angles to one another.

It is conceivable that the micrometer wires of the reading microscope may be set with smaller probable error on the central line of these narrow bands than on the centre of the ill-defined star image itself. It is also possible that the presence of a binary will be more readily perceived by the doubling of the bands than by observation of the confused disc itself, but I have not been able to try this yet. I shall be grateful if any astronomer can inform me if this method has been used for the purposes of measurement.

It may be noted that if a wire or a couple of parallel wires be placed between the object glass and eyepiece of a microscope, the focus of a star-image can be more readily determined than by observation of the ringed disc alone. The very fine narrow band of diffracted light crossing the centre of the disc passes more rapidly in and out of focus than can be observed with the central spot of the disc itself.

Imperial College of Science, London,

Alan Pollard.

Feb. 3.

Altered Character in the White-faced Spanish Fowl.

A RUMOUR gained currency last year that the old white-faced Spanish fowl, made classical by Darwin's experiments, was extinct. This is not so-a cock won the first prize in the "any other variety" class at the last Crystal Palace Poultry Show-but it is now very rare. Thus it seems to me worth mentioning that its characteristic points have undergone a great additional development since Darwin's time. The ear-lobes were then already large, fully continuous with the similar white skin of the face, and confluent with the throat-skin behind the wattles, but they did not hang down so far as those. By the 'seventies they did so, in some specimens at any rate, but still retained their character as ear-lobes. This has now been entirely lost; they form but the lateral portions of a great white bib or horizontal dewlap, which extends an inch or two below the wattles, the throat-skin having been much developed in the downward direction also.

It is obvious that this alteration must have been effected by selection within the breed, as no outcross could have been used, no other breed having the white face. Thus we have here one structure definitely changed into another by selection of small variations in about half a century. The cock above mentioned is figured in Poultry for Dec. 30, 1927.

c/o Grindlay and Co.,

F. FINN.

54 Parliament Street, S.W.1, Feb. 5.

No. 3042, VoL. 121] 